\title{
Influence of sulfate on anion exchange membranes in reverse electrodialysis
}

\author{
Diego Pintossi ${ }^{1,2}$, Chieh-Li Chen $\mathbb{E}^{1}$, Michel Saakes ${ }^{1}$, Kitty Nijmeijer $\mathbb{E}^{2,3 凶}$ and Zandrie Borneman ${ }^{2,3}$
}

Reverse electrodialysis (RED) is a technology producing renewable energy from the mixing of river and seawater. In natural salinity gradients, multivalent ions are present, which lead to a reduced RED power output. Transport of multivalent ions against the concentration gradient and their trapping inside the membranes leads to a lower driving force and increased membrane resistance. The present work focuses on the effect of sulfate ions on anion exchange membranes in RED. A monovalent ion selective membrane ability to retain a higher open circuit voltage is offset by the higher resistance in the presence of sulfate, leading to losses in normalized power outputs $(-25 \%)$ comparable to a standard grade membrane. Longer term experiments revealed that membrane resistance increases over time. This study highlights the need to address uphill transport, resistance increase, and decreased permselectivity of anion exchange membranes in presence of multivalent ions.

npj Clean Water (2020)3:29; https://doi.org/10.1038/s41545-020-0073-7

\section{INTRODUCTION}

To reduce the impact of global warming and climate change, reducing greenhouse gas emission is a key target. To achieve this, clean energy sources are essential. One emerging renewable energy source is salinity gradient energy, which is the generation of electricity from the controlled mixing of solutions with different salt contents ${ }^{1}$. To harvest salinity gradient energy, reverse electrodialysis (RED) is a promising technology ${ }^{2}$. Salinity gradients naturally occur where rivers meet the sea, but artificial sources such as desalination brines were also investigated ${ }^{3-5}$. Alternative applications of RED include energy storage systems and waste heat engines based on thermolytic salt gradients ${ }^{6-11}$.

In RED, anion exchange membranes (AEMs) and cation exchange membranes (CEMs) are stacked alternately to create feedwater channels where the high and low concentration solutions flow ${ }^{12}$. The concentration gradient across each membrane generates a voltage, which is the electromotive force to drive a current through the $\operatorname{stack}^{13}$. To power an external load, redox reactions at the two ends of the stack convert the ionic current flowing through the membranes into an electrical current (Fig. 1 $)^{14}$. When harvesting energy from natural salinity gradients, the power output of RED depends on the extent of the salinity gradient and the composition of the feedwaters. Clay particles, multivalent ions, natural organic matter, and micro-organisms in the feedwaters induce negative interactions with the membranes and clogging of the channels ${ }^{15}$. These interactions are referred to as fouling and cause the power generated by the RED stack to decrease over time. In particular, multivalent ions present a significant challenge because they affect power generation by simultaneously decreasing the available electromotive force and increasing the membrane resistance. The first effect results from uphill transport of multivalent ions from the river to the seawater side, while the second results from trapping of multivalent ions inside the membranes (Supporting Fig. 1).

Uphill transport results from the different electromotive force for monovalent and multivalent ions ${ }^{16,17}$. These two gradients act like DC generators connected in parallel ${ }^{18}$. The generator with the highest voltage spends energy to charge the one with the lowest voltage until they reach an equilibrium. Inside the RED stack, this means that the monovalent ion potential is decreased to increase the extent of the multivalent ion electromotive force. This transport is electroneutral, with two monovalent ions exchanged for each divalent ion. Therefore, no net current is produced and no energy is harvested in the uphill transport process ${ }^{18}$. The net result is a lower available electromotive force.

In addition to the lower voltage, multivalent ions also negatively affect the membrane properties. A multivalent ion entering an ion-exchange membrane (IEM) may (1) be transported through the membrane, (2) bind to a single fixed charge group, or (3) bind to multiple fixed charge groups in the membrane (Supporting Fig. 1b). If (1) occurs, the electrical resistance for multivalent ions transport is higher than for monovalent ions, therefore a higher ohmic drop is measured. If (2) happens, the multivalent ion reverses the charge of the fixed group and decreases the ion exchange capacity of the membrane, which in turn decreases its permselectivity and increases its resistance ${ }^{19}$. If (3) takes place, the multivalent ion neutralizes two fixed charged groups in the membrane and is trapped, causing a higher membrane resistance over time due to the lower ion exchange capacity of the membrane and the decreased free volume available for transport of other ions ${ }^{20}$.

Studies on the effects of multivalent ions on RED performance and strategies to mitigate them focused mainly on cations, i.e., mixtures of multivalent cations and anions ${ }^{16,18}$ or on multivalent cations $^{21,22}$. The lack of focus on multivalent anions was justified by the assumption that the negative effect of multivalent anions is limited due to the smaller hydrated radii of multivalent anions and the hypothesized presence of sulfate as a monovalent species $\left(\mathrm{NaSO}_{4}{ }^{-}\right)^{18,22}$. However, Rijnaarts et al. recently reported a study on $\mathrm{AEM}$ fouling in natural conditions ${ }^{23}$. Their results prove that organic fouling alone cannot justify the measured loss in power density, thus highlighting the need to investigate the negative

\footnotetext{
${ }^{1}$ Wetsus, European Centre of Excellence for Sustainable Water Technology, P.O. Box 1113, 8900 CC, Leeuwarden, the Netherlands. ${ }^{2}$ Membrane Materials and Processes, Department of Chemical Engineering and Chemistry, Eindhoven University of Technology, P.O. Box 513, 5600 MB, Eindhoven, the Netherlands. ${ }^{3}$ Dutch Institute for Fundamental Energy Research (DIFFER), P.O. Box 6336, $5600 \mathrm{HH}$, Eindhoven, the Netherlands. ${ }^{凶}$ email: d.c.nijmeijer@tue.nl
} 


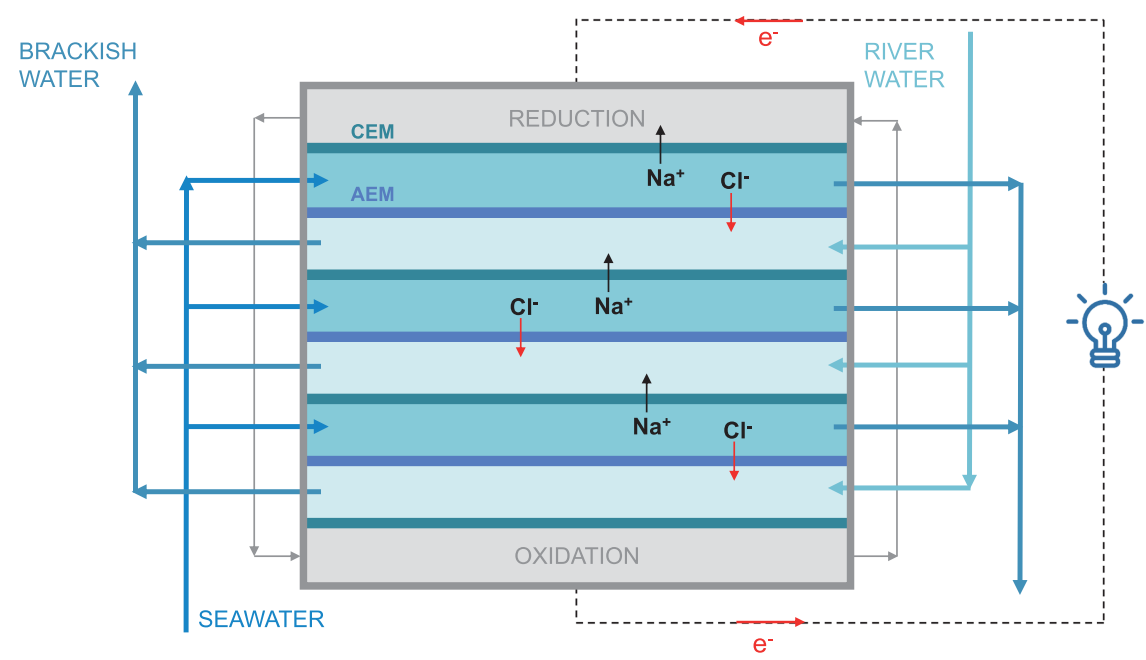

Fig. 1 Reverse electrodialysis stack. Schematic representation of a reverse electrodialysis stack. The repeating unit consists of a CEM, seawater compartment (with spacer), an AEM, and a river water compartment (with spacer).

Table 1. Measured membrane properties of the AEM used in the study.

\begin{tabular}{lllll}
\hline Membrane & Grade & Electrical resistance $(0.5 \mathrm{M} \mathrm{NaCl})$ & Permselectivity $(0.1 / 0.5 \mathrm{M} \mathrm{NaCl})$ & Water content \\
\hline Fujifilm AEM type I & Standard & Low $\left(1.46 \pm 0.11 \Omega \mathrm{cm}^{2}\right)$ & Low $(88.7 \%)$ & High $(50 \%)$ \\
Fujifilm AEM type 10 & Standard & Medium $\left(1.89 \pm 0.05 \Omega \mathrm{cm}^{2}\right)$ & Medium $(93.2 \%)$ & High $(41 \%)$ \\
Neosepta ACS & Monovalent ion selective & High $\left(4.49 \pm 0.12 \Omega \mathrm{cm}^{2}\right)$ & Medium (94.3\%) & Low $(26 \%)$ \\
Neosepta AMX-fg & Standard & High $\left(3.82 \pm 0.15 \Omega \mathrm{cm}^{2}\right)$ & High (95.5\%) & Low $(21 \%)$ \\
Neosepta CMX-fg & Standard & High $\left(3.14 \pm 0.11 \Omega \mathrm{cm}^{2}\right)$ & High (99.0\%) & Low (32\%) \\
\hline
\end{tabular}

impact of other forms of fouling on AEMs, including multivalent anions.

This work thus investigates and elucidates the effect of sulfate on AEMs in RED. Testing of standard grade and monovalent ionselective membranes was carried out with different sulfate fractions in the feedwaters. Stack and membrane characterizations were used to quantify the impact of sulfate on the open-circuit voltage (OCV), electrical resistance, and power output. The results of this systematic study of the effect of multivalent anions on AEMs in RED contribute to (1) quantifying the differences between fouling due to multivalent anions and cations, (2) understanding fouling in natural conditions, and (3) guiding future AEM development.

\section{RESULTS AND DISCUSSION}

To evaluate the impact of sulfate on RED performance, the following stack properties for four different membrane types in the presence or absence of sulfate were monitored: OCV, stack electrical resistance, and gross power density. In addition, the effect of sulfate ions on the membrane properties (electrical resistance and permselectivity) was investigated.

The four different AEM membranes used are Fujifilm type I (low electrical resistance, low permselectivity, high water content), Fujifilm type 10 (medium electrical resistance, medium permselectivity, high water content), Neosepta ACS (a monovalent ion selective membrane with a high electrical resistance, medium permselectivity, low water content), Neosepta AMX-fg (high electrical resistance, high permselectivity, low water content). These membranes are selected because they cover a broad range of the most essential properties relevant for power generation in RED. Details of the membranes are given in Table 1.

The experimental results are compared to a model calculating the OCV in the presence of multivalent ions in one or in both of the feedwaters (developed by Vermaas et al. and used as reported, further information in the "Methods" section) $)^{9}$. This model is based on the equilibration between the electromotive force of monovalent and multivalent ions when increasing amounts of multivalent ions in the river water are exchanged for monovalent ions in the seawater.

\section{OCV and uphill transport}

Figure 2 shows the normalized OCV as a function of the sulfate fraction $(10,25$, and $50 \mathrm{~mol} . \%)$ present in the feedwaters (both sides, river water only and seawater only), measured in a stack experiment. Supporting Table 1 in the supporting information reports the OCV values prior to normalization.

Figure 2a illustrates the results of the stack experiments with equivalent fractions of sulfate on both sides, river, and seawater. The normalized OCV decreases for all membranes in accordance with the model. The monovalent ion selective ACS membrane (high electrical resistance, medium permselectivity, and low water content) outperforms the model, while the standard grade membranes all exhibit similar losses of OCV and perform worse than the model, independent of their properties. The monovalent ion selective membrane ACS limits sulfate transport; therefore, uphill transport is hindered and the stack is able to operate at higher OCV than predicted by the model. Nevertheless, at increasing fractions of sulfate in the feedwaters, also ACS increasingly allows sulfate transport, leading to a decrease in normalized OCV, albeit over the full range less than for the other membranes. The deviation from the model of the standard grade membranes is attributed to a loss in permselectivity induced by the presence of sulfate ions. This explanation is supported by the membrane permselectivity measurements using mixtures of $\mathrm{NaCl}$ and $\mathrm{Na}_{2} \mathrm{SO}_{4}$ (Fig. 3a, Supporting Table 2). The loss in permselectivity is larger for the type I (low electrical resistance, low 

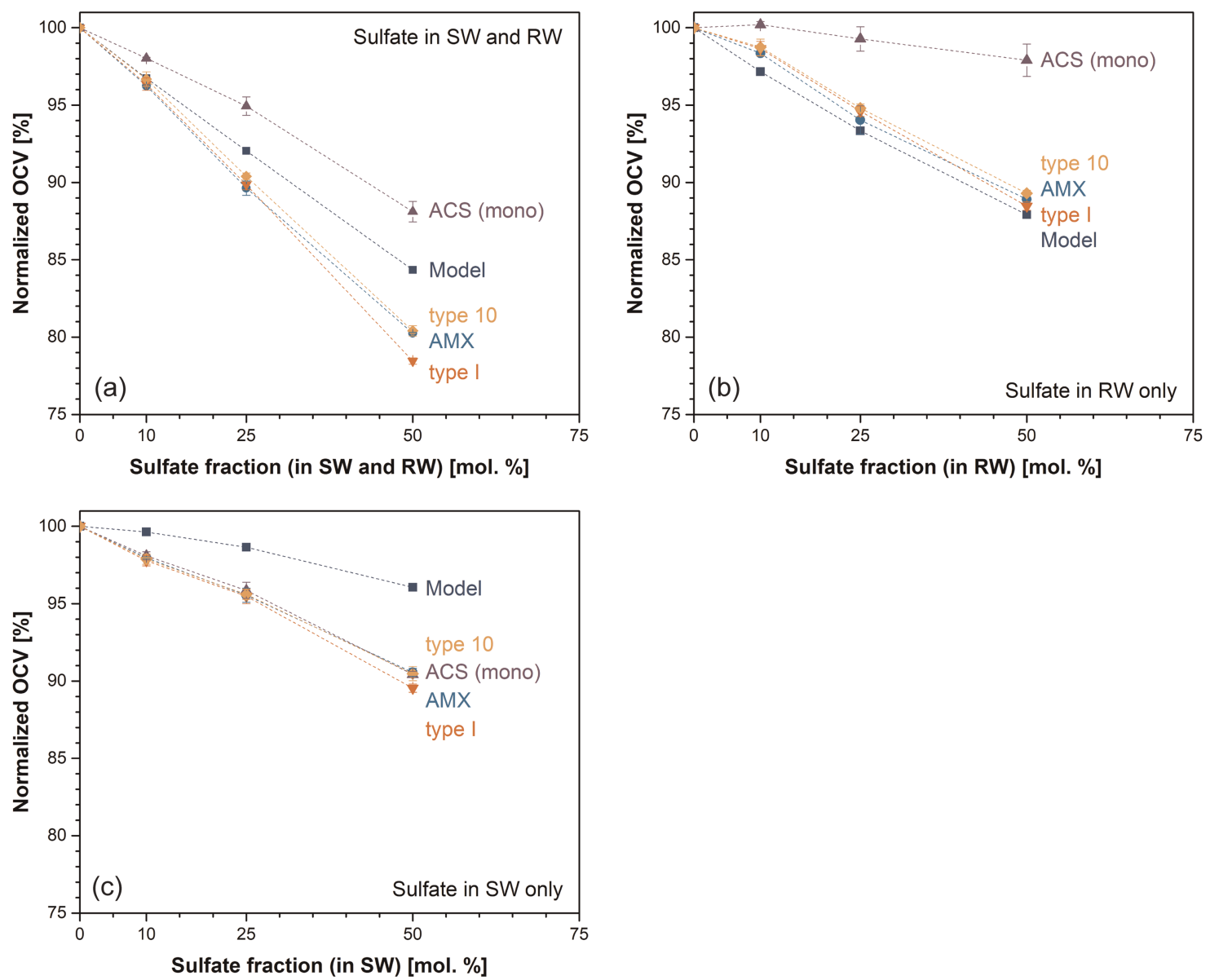

Fig. 2 Normalized OCV versus sulfate fraction. Normalized OCV versus sulfate fraction for the scenarios with (a) equal amounts of sulfate in both feedwaters, (b) sulfate present in the river water only, and (c) sulfate present in the seawater only. SW seawater; RW river water. Results have been normalized to the values measured with clean waters (before introducing multivalent anions in the feedwaters). All experiments were performed in duplicate with error bars illustrating the minimum and maximum points.

permselectivity, and high water content), which is the membrane with the lowest starting permselectivity and the highest swelling degree. Similar results were reported for CEMs and multivalent cations by Rijnaarts et al. and Moreno et al. ${ }^{21,22}$. Permselectivity and its decrease due to sulfate presence are not accounted for in the model, explaining the discrepancy between the model and the experimental results.

Figure $2 \mathrm{~b}$ illustrates the scenario with sulfate ions only present in the river water. In this case uphill transport plays a role. The loss in normalized OCV is not as severe as for the scenario with sulfate ions on both sides, but quite significant though. The amount of sulfate ions that the membranes experience is lower due to the low absolute amount of sulfate ions in the river water. Therefore, the monovalent ion-selective membrane is able to retain its full $\mathrm{OCV}$, and the loss in permselectivity for the standard grade membranes is limited as well. It can be noted that the standard grade membrane data lie slightly above the model. This is the case because the predicted batch model OCV is attained at a very low sulfate ion fraction (Supporting Fig. 2b in Supporting Information), which cannot be reached in the stack experiments since fresh solutions are continuously supplied. Despite this limitation, the model still provides a close estimation of the OCV with sulfate present in river water only.

The last scenario, with sulfate ions only present in the seawater, is visualized in Fig. 2c. Unlike the other two scenarios, uphill transport of sulfate ions is not possible and the only species that are transported against their gradient are chloride ions.
Nevertheless, the increase in OCV due to chloride uphill transport is balanced by the decreased chloride gradient. Thus, the overall effect is a reduced OCV at increasing fractions of sulfate for all membranes. Membrane permselectivity decreases due to the large amount of sulfate ions present in the seawater, leading to deviations from the behavior predicted by the model. The loss of permselectivity observed at the stack level for Neosepta ACS seems to contradict the permselectivity values obtained at the membrane level (Fig. 3a). This is explained by taking into account the slow access (diffusion limitation) of sulfate ions to the membrane, which are equilibrated at a low sulfate fraction (Supporting Table 3).

To further verify the occurrence of uphill transport, the outlet feedwater composition was analyzed. Figure $3 \mathrm{~b}$ illustrates the results of the ion chromatography (IC) analyses.

The lower concentration of sulfate in the river water at the outlet than at the inlet during the constant current step confirms the movement of multivalent anions from river to sea. This is especially visible for the two membranes with lower permselectivity and higher water content (type I and type 10), as these are much more permeable for the bivalent sulfate ions than especially the monovalent ion selective ACS membrane, which keeps the highest river water sulfate concentration over the full duration of the process. Additionally, in the third scenario (sulfate in seawater only), sulfate is found in the river water outlet, showing its transport downhill as a consequence of the applied current, which 

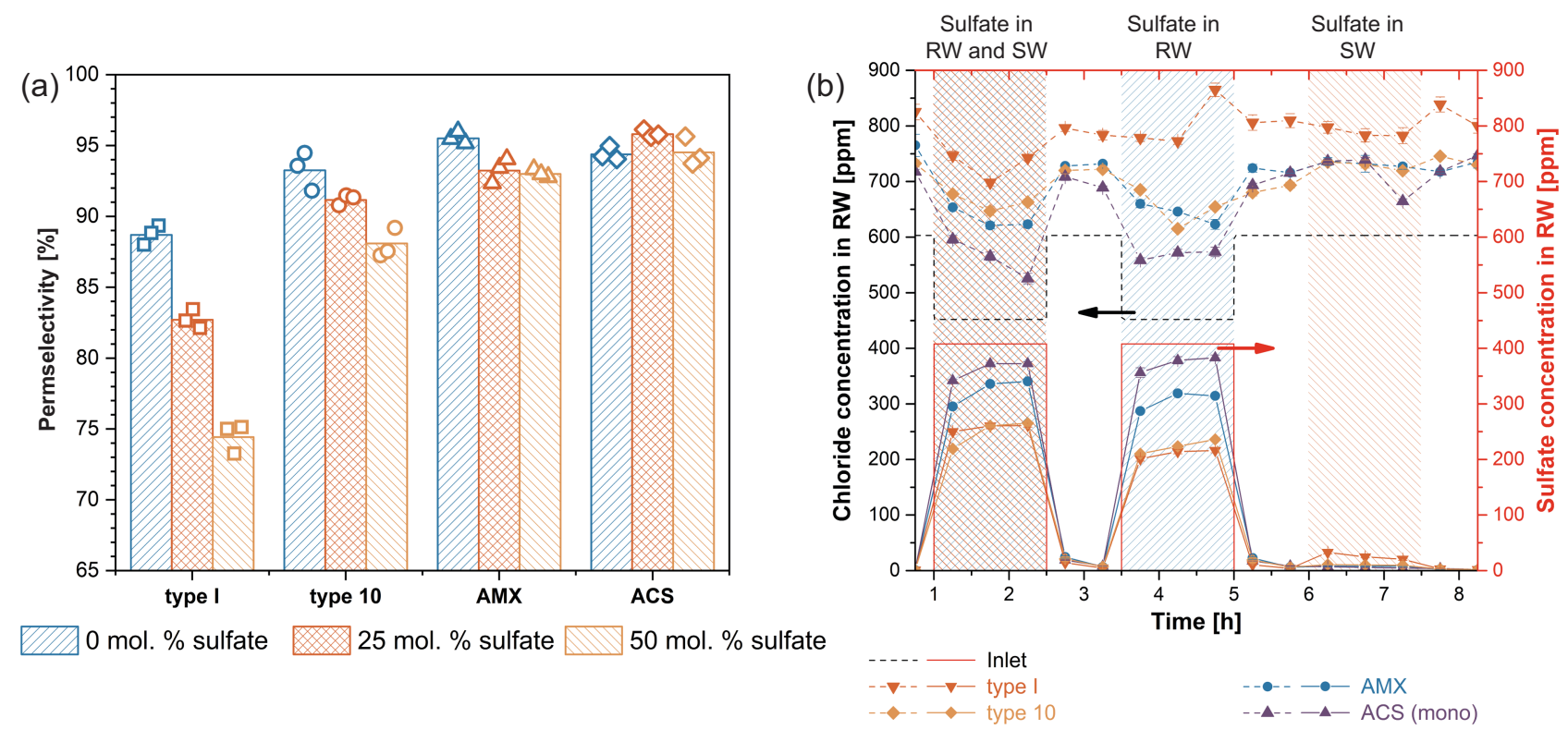

Fig. 3 Membrane permselectivity and ion concentrations at the stack outlets. (a) apparent permselectivity of the four AEMs at a fraction of 0,25 , and $50 \mathrm{~mol} . \%$ sulfate and the remaining part chloride ions, measured for a single membrane. Measurements were performed in triplicate, with all experimental points shown as dot plots, while bars illustrate average values. $\mathbf{b}$ In- and outlet chloride and sulfate concentrations in the river water during the constant current step for the stack experiments with $25 \mathrm{~mol} . \%$ sulfate. The solid lines represent the sulfate concentrations while the dotted lines represent the chloride concentrations. The black and red line are the concentration of chloride and sulfate respectively supplied at the stack inlet (feed). The symbols represent the corresponding concentrations measured at the stack outlet for the different membranes. The low sulfate concentrations measured in the third scenario (sulfate in seawater) for the four membranes are significant compared to control samples. Experiments were performed in duplicate and each water samples was analyzed in duplicate, with error bars illustrating the minimum and maximum value.

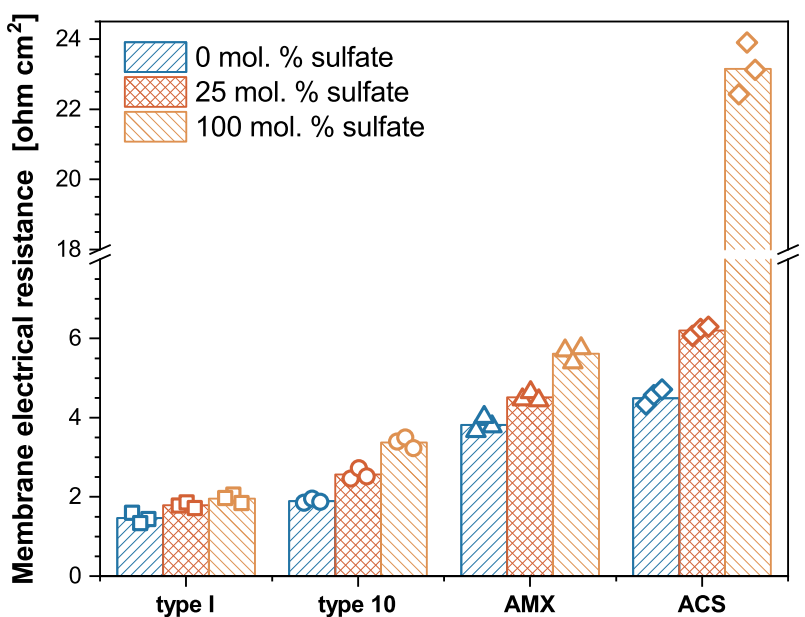

Fig. 4 Membrane electrical resistance membrane. Electrical resistance of the investigated $\mathrm{AEMs}$ in $100 \mathrm{~mol} \%$ sodium chloride $(0.5 \mathrm{M})$, a mixture of $75 \mathrm{~mol} . \%$ sodium chloride $(0.375 \mathrm{M})$ and $25 \mathrm{~mol} . \%$ sodium sulfate $(0.125 \mathrm{M})$, and $100 \mathrm{~mol} . \%$ sodium sulfate $(0.5 \mathrm{M})$, measured in a six-compartment cell. Measurements were performed in triplicate, with all experimental points shown as dot plots, while bars illustrate average values.

is carried by both chloride and sulfate, and the uphill transport of chloride.

\section{Electrical resistance}

Figures 4 and 5 illustrate the membrane electrical resistance and the stack electrical resistance respectively measured in the presence of increasing fractions of sulfate in the feedwaters.

The transport selectivity for chloride over sulfate of an AEM is expressed as the ratio between its resistance measured in $\mathrm{NaCl}$ only and its resistance measured in $\mathrm{Na}_{2} \mathrm{SO}_{4}$ only ${ }^{22}$. The membrane electrical resistance increases for all AEMs in the presence of sulfate. The monovalent ion selective ACS exhibits a $\mathrm{Cl}^{-} / \mathrm{SO}_{4}{ }^{2-}$ selectivity equal to 5.15 , thus confirming its preferential transport of monovalent ions, which is granted by its high crosslinking degree, allowing for size exclusion of multivalent ions. The standard grade membranes are more permeable to sulfate and their $\mathrm{Cl}^{-} / \mathrm{SO}_{4}{ }^{2-}$ selectivities range between 1.34 and 1.78 , with type 10 (medium electrical resistance, medium permselectivity, and high water content) and type I (low electrical resistance, low permselectivity, and high water content) having a low selectivity due to their high water content (Table 1), while AMX (high electrical resistance, high permselectivity, and low water content) compensates its low water content with a high charge density ${ }^{23}$.

The evolution of the stack electrical resistance (Fig. 5, measured as the slope of the current-voltage plot, without blank correction) is consistent with the measurements at the membrane level. Figure 5a shows the scenario with sulfate present in both sea and river water. Although the overall stack resistance is decreasing due to the increased water conductivity and this is included in the model calculations, the experimental data in Fig. 5 are positioned still well above the model values, showing an increased AEM resistance. The stack with the monovalent ion selective ACS has almost constant electrical resistance as it is designed to reduce sulfate transport. Hence, the increase in AEM resistance (Fig. 3) balances the increased water conductivity. On the contrary, the standard grade membranes are more permeable to sulfate and are positioned slightly above the model, with a small AEM electrical resistance increase, as measured at the membrane level (Fig. 4).

Similar behavior is observed for Fig. $5 b$, with sulfate in the river water only. In Fig. 5c, with sulfate in the seawater only, there is no increase in the conductivity of the river water, which is the main component of the stack electrical resistance. Therefore, the effect of sulfate on the stack electrical resistance is less evident. 

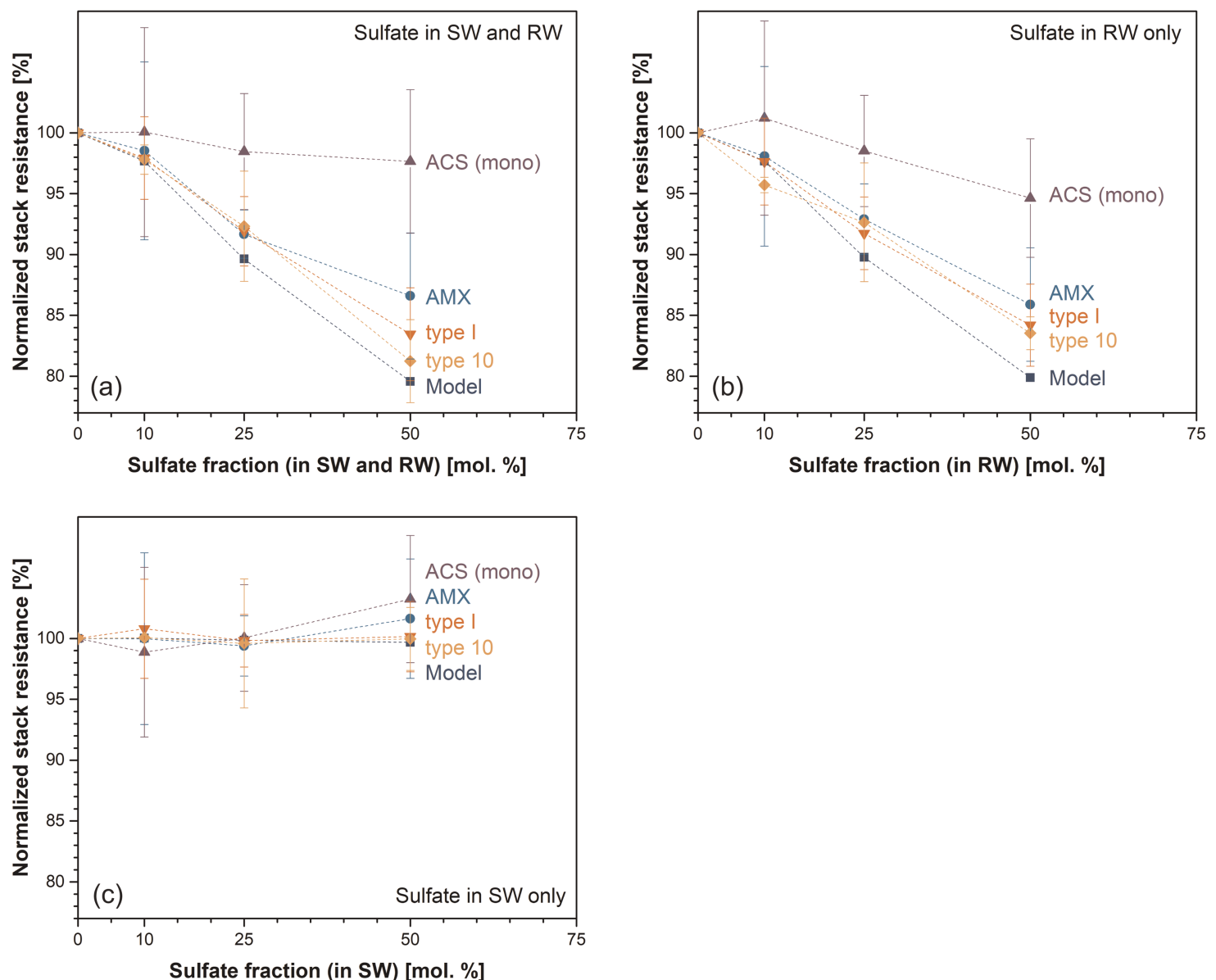

Fig. 5 Normalized stack electrical resistance versus sulfate fraction. Normalized stack electrical resistance versus sulfate fraction (a) with sulfate in both feedwaters, (b) with sulfate present on the river side only, and (c) with sulfate present on the sea side only. SW seawater; RW river water. Results have been normalized to the values measured with clean waters (before introducing multivalent anions in the feedwaters). All experiments were performed in duplicate with error bars illustrating the minimum and maximum points.

Table 2. Sequence of the feedwaters compositions for RED stack experiments. The sulfate fraction $\mathrm{x}$ was equal to 10,25 , and 50 mol.\% in three sets of experiments.

\begin{tabular}{|c|c|c|c|c|c|c|c|}
\hline Time [h] & $0-1$ & $1-2.5$ & $2.5-3.5$ & $3.5-5$ & $5-6$ & $6-7.5$ & $7.5-8.5$ \\
\hline \multicolumn{8}{|l|}{ Sea } \\
\hline $\mathrm{NaCl}$ [mol.\%] & 100 & $100-x$ & 100 & 100 & 100 & $100-x$ & 100 \\
\hline $\mathrm{Na}_{2} \mathrm{SO}_{4}$ [mol.\%] & 0 & $\mathrm{x}$ & 0 & 0 & 0 & $x$ & 0 \\
\hline $\mathrm{NaCl}$ [mol.\%] & 100 & $100-x$ & 100 & $100-x$ & 100 & 100 & 100 \\
\hline $\mathrm{Na}_{2} \mathrm{SO}_{4}$ [mol.\%] & 0 & $\mathrm{x}$ & 0 & $x$ & 0 & 0 & 0 \\
\hline
\end{tabular}

Nevertheless, at high sulfate fractions, ACS (monovalent ion selective) still shows the highest resistance increase as it is designed to limit sulfate transport.

The negative effect of sulfate on the AEM resistance is limited compared to the effect of multivalent cations on $\mathrm{CEMs}^{21,22}$ due to the smaller hydrated radius and hydration free energy of sulfate compared to magnesium and calcium (see Feedwaters in Methods). Comparing the results presented here with those of Moreno et al. for standard grade membranes, at the same molar fractions of multivalent ions (10 and $25 \mathrm{~mol} . \%$ ), the increase in electrical resistance for the CEMs compensates the increased electrical conductivity of the feedwaters, leading to an increased stack resistance. For the AEMs that is not the case, with an overall decrease in stack resistance despite the increased membrane resistance (Figs 4 and 5).
Figure 6 shows the evolution over time of the stack electrical resistance when exposing ACS or type 10 first to $\mathrm{NaCl}$ only, followed by $25 \mathrm{~mol} . \%$ of sulfate on both sides and subsequently $\mathrm{NaCl}$ only again. When switching from $\mathrm{NaCl}$ to a mixture containing sulfate (at $1 \mathrm{~h}$ in the plot), the stack electrical resistance drops initially due to the increased water conductivity, then it increases slowly due to the trapping of sulfate in the AEM. The opposite happens when switching back to waters with only $\mathrm{NaCl}$ (at $2 \mathrm{~h}$ in the plot). This shows that the change in membrane resistance is not instantaneous due to limitations in ion transport rate, while the effect of water conductivity has an immediate effect on stack resistance. Interestingly, the evolution of stack resistance over time for the type 10 membrane (standard grade membrane) is different as the resistance initially drops due to the increased water conductivity and then plateaus (Fig. 6), which is 
due to the faster transport of sulfate through the standard grade membrane. These results differ from the finding of Moreno et al. for CEMs exposed to magnesium, as the electrical resistance of the standard grade CEMs used in their study increases over time when exposed to magnesium ${ }^{21}$. The standard grade AEMs used in our study behave similarly to the multivalent ion-permeable Fujifilm CEM T1 reported in the study of Moreno et al. due to the smaller hydrated radius and lower hydration energy of sulfate than magnesium (see Feedwaters in Methods) combined with the high water content (type I and 10) or charge density (AMX) of the standard grade AEMs used in our study.

This conclusion is also supported by the chloride over sulfate selectivity of the three standard grade AEMs investigated in our study, which is lower than 2 (which is comparable to the selectivity of sodium over magnesium of the multivalent ion-permeable Fujifilm CEM T1 ${ }^{22}$ ). Such a low $\mathrm{Cl}^{-} / \mathrm{SO}_{4}{ }^{2-}$ selectivity implies that the standard grade membranes transport multivalent ions almost in equal amounts as the monovalent ions. Therefore, sulfate trapping is less likely, which leads to the stable electrical resistance for type 10 in Fig. 6.

These different electrical resistance evolutions over time in the presence of multivalent anions or cations for AEMs and CEMs stem from the smaller hydrated radii and lower hydration energies of multivalent anions than cations ${ }^{24}$, which make the multivalent anions easier to transport than multivalent cations. Nevertheless, multivalent anions are still larger than monovalent ones ${ }^{24}$ and can be trapped inside the AEMs, covering the fixed membrane charges and causing an increase in electrical resistance. At the same time, multivalent anions are more difficult to selectively reject than their cationic counter parts since they span a smaller range of ionic radii and hydration energies (see Feedwaters in Methods) ${ }^{25}$. Epsztein et al. recently showed the role played by the ion hydration free energy in transport selectivity for ion exchange membranes ${ }^{26}$. The $\mathrm{Cl}^{-} / \mathrm{SO}_{4}{ }^{2-}$ selectivity of the monovalent ion selective Neosepta ACS is around 5, while the Neosepta CMS (monovalent ion selective CEM) exhibits a $\mathrm{Na}^{+} / \mathrm{Mg}^{2+}$ selectivity of $34^{22,27}$. While this benefits the standard grade AEMs in terms of limited electrical resistance increase in presence of multivalent ions, it is detrimental in terms of OCV loss.

Although the stack electrical resistance results reported above suggest that for standard grade membranes sulfate has a limited impact on stack resistance, these experiments were only conducted during one day. A longer experiment (11 days) was carried out to investigate the effect on the AEMs of longer exposure to sulfate. The results of this long term experiment

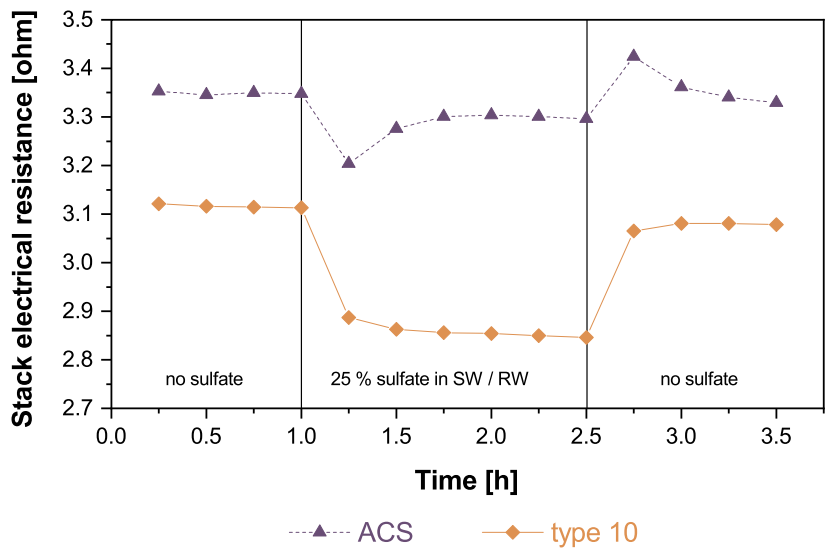

Fig. 6 Stack electrical resistance when changing feedwaters. Electrical resistance of the stack with ACS (monovalent ion selective membrane) and type 10 (standard grade) in the transients from $100 \mathrm{~mol} . \% \mathrm{NaCl}$ to the mixture with $25 \mathrm{~mol} . \% \mathrm{Na}_{2} \mathrm{SO}_{4}$ and back to the $100 \mathrm{~mol} \% \mathrm{NaCl}$ feedwaters. Data are reported for one experiment per membrane type.
(Supporting Fig. 4, Supporting Information) show that on the longer term, multivalent anions do have a negative impact on the resistance of both membranes, independent of the type and that monovalent ion-selective membranes are even more severely affected than standard grade membranes. Considering the mechanisms presented in Supporting Fig. $1 \mathrm{~b}$, the results from Supporting Figs. 4 and 6 show that sulfate trapping is relevant on longer-term experiments, while on the short term only the monovalent-ion selective ACS experiences a reversible increase in electrical resistance and all membranes experience permselectivity loss.

\section{Gross power density}

Figure 7 shows the evolution of gross power density in RED with increasing sulfate fractions for all membranes and the three exposure scenarios. The normalized gross power density is based on the OCV and stack electrical resistance (Eq. 3).

Figure 7a shows the results for the scenario with sulfate in both sea and river water. For all membrane types and the model, the normalized power density decreases with increasing sulfate fraction because the OCV decreases in presence of sulfate, while the stack electrical resistance increases and both are detrimental for gross power density. The model predicts a smaller loss in power density than measured experimentally, as the experimental loss in membrane permselectivity in the presence of sulfate is not accounted for in the model. Remarkably, the monovalent ion selective ACS membrane does not outperform the standard grade membranes. Despite retaining a higher OCV in presence of sulfate, its large increase in electrical resistance due to sulfate trapping and limited sulfate transport lowers its performance to a level comparable to that of the standard grade membranes. Among the standard grade membranes, the type 10 membrane outperforms type I and AMX, especially at high sulfate fractions, thanks to its combination of intermediate resistance and permselectivity. The other standard grade membranes analyzed, type I and AMX, perform slightly worse than the type 10. Type I has the benefit of a low resistance thanks to its high water content, but its low permselectivity is reflected in a larger OCV loss in the presence of sulfate. AMX has a high permselectivity but also a high electrical resistance. Since transport of sulfate through the $A M X$ is possible, the OCV loss and high electrical resistance limit its gross power density in the presence of sulfate. Figure $7 b$ shows the results for the scenario with sulfate only in the river water. In this case, the decrease in normalized power density is far less pronounced because the OCV decreases in presence of sulfate in river water, but the stack electrical resistance also decreases, due to its increased conductivity, and that is beneficial for the gross power density. The monovalent ion selective ACS membrane performs better than the model and the standard grade membranes thanks to its fully retained OCV, especially at high sulfate fractions. Type 10 performs better than the type I and AMX membrane as it combines a smaller OCV loss (type I loses more than type 10) with a smaller increase in electrical resistance (AMX increases more than type 10). Figure 7c illustrates the results of the scenario with sulfate only in the seawater. Here, again a steep decrease in normalized power density is observed for all membranes and the model because the partial substitution of sodium chloride with sodium sulfate in the seawater decreases the extent of the gradient and thus the OCV, which is detrimental for gross power density. Moreover, the decrease is equal for all membranes as the OCV loss is mainly due to the reduced extent of the sodium chloride gradient. Additionally, the conductivity of river water is not increased as sulfate is not present, therefore the changes in stack electrical resistance are comparable for the different membranes, which leads to similar losses in normalized gross power density for all membranes. 

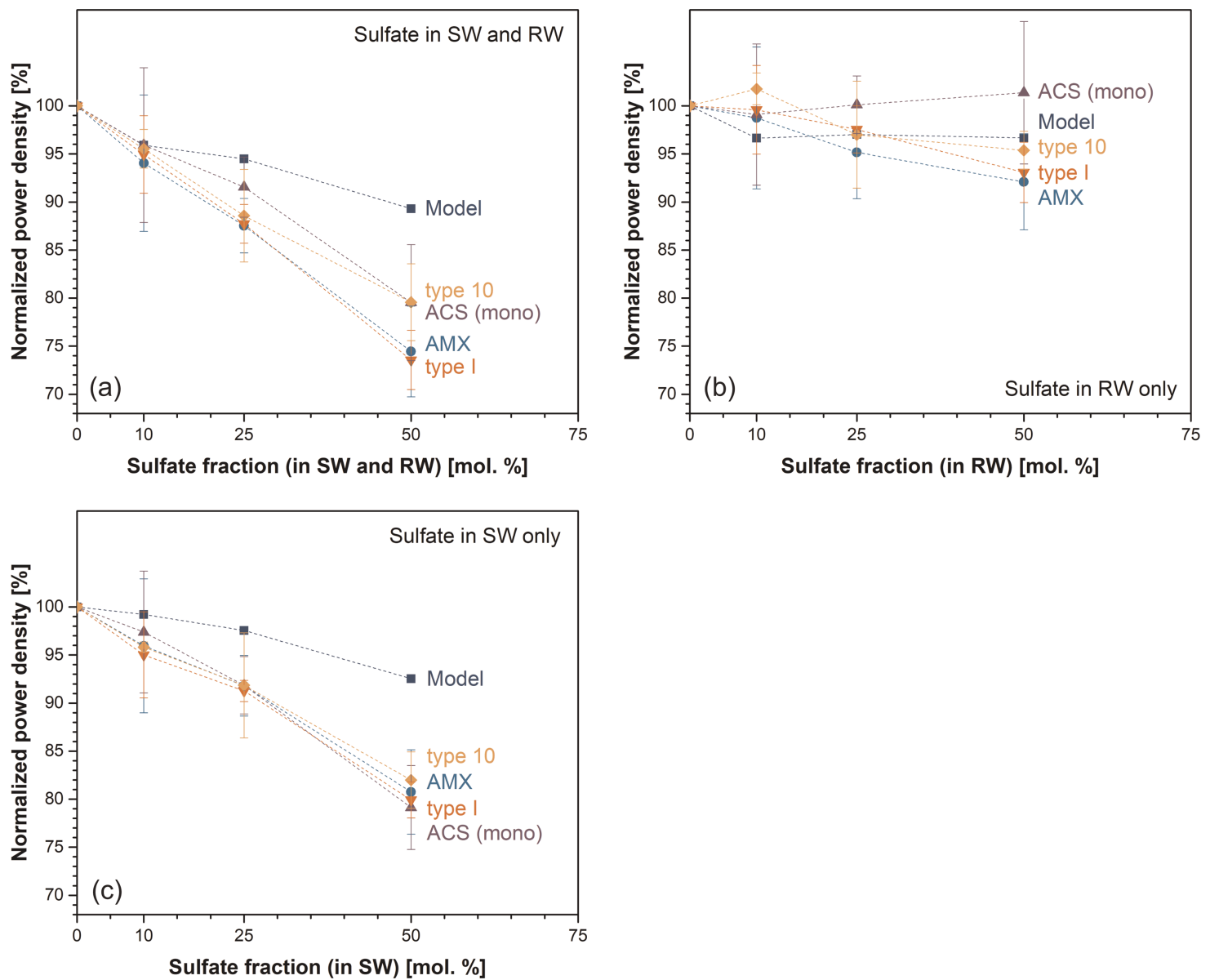

Fig. 7 Normalized gross power density versus sulfate fraction. Normalized gross power density versus sulfate fraction for the scenario with (a) sulfate in both feedwaters, (b) sulfate present in the river water only, and (c) sulfate present in the seawater only. SW seawater; RW river water. Results have been normalized to the values measured with clean waters (before introducing multivalent anions in the feedwaters). All experiments were performed in duplicate with error bars illustrating the minimum and maximum points.

Our experiments on the effect of multivalent anions on AEMs in RED with standard grade and monovalent ion selective membranes show that sulfate deteriorates RED performance due to the decreased OCV resulting from uphill transport and loss in membrane permselectivity, and increased membrane resistance, which increases during long term exposure to the multivalent anions. While these effects of multivalent anions are similar to those of multivalent cations, the narrower range of hydrated radii and hydration energies for anions results in a smaller electrical resistance increase over time, but also in lower monovalent-ion selectivities, which are detrimental for the OCV. When sulfate is present on both sides of the membranes, for the standard grade membranes gross power density losses up to $25 \%$ are measured. Similar losses can be expected also for other multivalent anions based on their hydrated radii and hydration energies. As an example, phosphate has been found to severely increase the electrical resistance of AEMs, while decreasing membrane permselectivity in ED processes ${ }^{28}$. Monovalent ion selective membranes can reduce the uphill transport of multivalent ions, but they do so at the expense of high electrical resistance, which increases even further in the presence of sulfate. Therefore, the normalized power density of the monovalent ion selective ACS is not higher than that obtained with standard grade membranes. Although they are subject to the negative effect of uphill transport and loss in permselectivity in the presence of sulfate, standard grade membranes have a limited increase in resistance only, in presence of multivalent anions. Thanks to its intermediate resistance and permselectivity, the standard grade type 10 membrane can match the performance of the monovalent ion selective ACS membrane. Nevertheless, when exposed to high sulfate fractions for a long time, both monovalent ion selective and standard grade membranes suffer from an increased electrical resistance. The results of the present study highlight the need to consider the negative effect of multivalent anions when developing new membranes that find a balance between RED performance and fouling. For sulfate, the main mechanisms to be addressed are: (1) uphill transport, (2) decreased permselectivity and (3) long term increase in membrane resistance in the presence of multivalent anions.

\section{METHODS}

$\mathrm{OCV}$ and uphill transport theory

The OCV is the voltage measured at the electrodes of a RED stack when no current is flowing through it. In the case of a $\mathrm{NaCl}$ gradient, it can be evaluated with the modified Nernst equation ${ }^{29}$ :

$$
\begin{aligned}
E & =a_{C E M} \frac{R T}{Z F} \ln \left(\frac{\gamma_{S W, N a^{+}} C_{S W, N a^{+}}}{\gamma_{R W, N a^{+}} C_{R W, N a^{+}}}\right)+a_{A E M} \frac{R T}{z F} \ln \left(\frac{\gamma_{S W, C l} C_{S W, C l}}{\gamma_{R W, C l} C_{R W, C l}}\right) \\
& =2 a_{a v g} \frac{R T}{z F} \ln \left(\frac{\gamma_{S W, N a C l} C_{S W, N a C l}}{\gamma_{R W, N a C l} C_{R W, N a C l}}\right)
\end{aligned}
$$

where $E$ is the electromotive force $[\mathrm{V}], a_{C E M}$ is the permselectivity of the CEM [-], $a_{A E M}$ is the permselectivity of the AEM [-], $R$ is the universal gas constant $\left[\mathrm{J} \mathrm{mol}^{-1} \mathrm{~K}^{-1}\right], T$ is the absolute temperature $[\mathrm{K}], z$ is the valence of 
the ions $[-], F$ is the Faraday constant $\left[\mathrm{C} \mathrm{mol}^{-1}\right], \gamma$ is the molar activity coefficient, $c$ is the molar concentration $\left[\mathrm{mol} \mathrm{L}^{-1}\right]$, and $a_{\text {avg }}$ is the average membrane permselectivity [-].

When considering mixtures of monovalent and multivalent ions, like chloride and sulfate, the electromotive force for chloride is higher than that of sulfate, because of the higher valence and lower activity coefficients of sulfate than of chloride. The difference between the two potentials acts as driving force to move sulfate from river to sea (against the concentration gradient, hence uphill), while moving chloride from sea to river (downhill). In this process, the gradient of sulfate increases and so does its potential, while the opposite happens for chloride. Such process, known as uphill transport, goes on until the two potentials are equilibrated and there is no driving force available for the exchange. The result is a lower OCV than the one predicted for the chloride gradient alone. Vermaas et al. presented a model to evaluate the OCV after uphill transport for perfectly permselective membranes in batch conditions ${ }^{18}$. The model developed by Vermaas et al. was used in this work as reported, only applied to multivalent anions.

It consists of two water compartments separated by an AEM (Vermaas presented the case with a CEM). On one side, a high concentration salt mixture is present. On the other side, there is a low concentration salt mixture. The considered salts for uphill transport across an AEM are sodium chloride and sodium sulfate.

The potential of the monovalent (e.g. chloride) and multivalent (e.g. sulfate) salts can be described by the modified Nernst equation (membrane permselectivity is assumed to be $100 \%$ ):

$E_{C I^{-}}=\frac{R T}{F} \ln \left(\frac{\gamma_{S W, C I^{-}} C_{S W, C C^{-}}}{\gamma_{R W, C^{-}} C_{R W, C C^{-}}}\right)$

$E_{\mathrm{SO}_{4}^{2-}}=\frac{R T}{2 F} \ln \left(\frac{\gamma_{S W, \mathrm{SO}_{4}^{2-}-} C_{S W, \mathrm{SO}_{4}^{2-}}}{\gamma_{\mathrm{RW}, \mathrm{SO}_{4}^{2-}} C_{\mathrm{RW}, \mathrm{SO}_{4}^{2-}}}\right)$

Uphill transport terminates when the two potentials are equilibrated, which yields the following condition for the concentration of the ions on both sides:

$$
\left(\frac{\gamma_{S W, C I^{-}} C_{S W, C I^{-}}}{\gamma_{R W, C C^{-}} C_{R W, C C^{-}}}\right)=\left(\frac{\gamma_{S W, \mathrm{SO}_{4}^{2-}} C_{S W, \mathrm{SO}_{4}^{2-}}}{\gamma_{\mathrm{RW}, \mathrm{SO}_{4}^{2-}} C_{\mathrm{RW}, \mathrm{SO}_{4}^{2-}}}\right)^{\frac{1}{2}}
$$

The molar amount of sulfate travelling uphill, $J_{\text {up,eq }}\left[\mathrm{mol} \mathrm{L}^{-1}\right]$ can be found by solving the following equation, derived from Eq. 4:

$$
\left(\frac{\gamma_{S W, C I^{-}}\left(c_{S W, C I^{-}}^{0}-2 J_{u p}\right)}{\gamma_{R W, C I^{-}}\left(c_{R W, C I^{-}}^{0}+2 \nu_{u p}\right)}\right)=\left(\frac{\gamma_{S W, S O_{4}^{2-}}\left(c_{S W, S O_{4}^{2-}}^{0}+J_{u p}\right)}{\gamma_{R W, S O_{4}^{2-}}\left(c_{R W, S O_{4}^{2-}}^{0}-J_{u p}\right)}\right)^{\frac{1}{2}}
$$

The activity coefficients are assumed to be constant (for small changes in concentration). Supporting Fig. 2 shows the graphical solution of Eq. 5. At equilibrium, the OCV for monovalent and multivalent ions is equal to:

$$
\begin{aligned}
O C V=E_{C I^{-}}=E_{S O_{4}^{2-}} & =\frac{R T}{F} \ln \left(\frac{\gamma_{S W, C I^{-}}\left(c_{S W, C I^{-}}^{0}-2 J_{u p, e q}\right)}{\gamma_{R W, C I^{-}}\left(c_{R W, C I^{-}}^{0}+2 J_{u p, e q}\right)}\right) \\
& =\frac{R T}{2 F} \ln \left(\frac{\gamma_{S W, S O_{4}^{2-}}\left(c_{S W, S O_{4}^{2-}}^{0}+J_{u p, e q}\right)}{\gamma_{R W, S O_{4}^{2-}}\left(c_{R W, S O_{4}^{2-}}^{0}-J_{u p, e q}\right)}\right)
\end{aligned}
$$

\section{Stack electrical resistance theory}

The ohmic component of the stack electrical resistance can be described by the following equation ${ }^{30}$ :

$$
R_{\text {stack }}=R_{e l}+\frac{N}{A}\left(R_{C E M}+R_{A E M}+\frac{1}{f} \frac{d_{R W}}{\kappa_{R W}}+\frac{1}{f} \frac{d_{S W}}{\kappa_{S W}}\right)
$$

where $R_{\text {stack }}$ is the stack electrical resistance $[\Omega], R_{e l}$ is the electrode resistance $[\Omega]$ (less relevant for increasing number of cell pairs), $N$ is the number of cell pairs [-], $A$ is the active membrane area $\left[\mathrm{cm}^{2}\right], R_{C E M}$ and $R_{A E M}$ are the areal resistance of CEM and AEM $\left[\Omega \mathrm{cm}^{2}\right], f$ is the obstruction factor [-] accounting for the presence of non-conductive spacers in the feedwater channels, $d$ is the compartment thickness $[\mathrm{cm}]$, and $K$ is the conductivity of the feedwaters $\left[\mathrm{S} \mathrm{cm}^{-1}\right]$. It is worth noting that substituting equimolar amounts of monovalent salt in the feedwater with multivalent salt will have a detrimental effect on the membrane resistance, but at the same time it will result in increased water conductivity, which is beneficial for the overall stack resistance.

\section{Gross power density}

The gross power density of a RED stack is calculated as the ratio between the maximum power point and the total membrane area of the stack. The maximum power point is achieved when the stack is working at half of the OCV, powering a load that is equal to the stack electrical resistance ${ }^{31}$. Therefore, the gross power density is given by:

$P_{\text {dens,gross }}=\frac{1}{2 N A} \frac{O C V^{2}}{4 R_{\text {stack }}}$

where $P_{\text {dens }}$ is the gross power density $\left[\mathrm{W} \mathrm{m}^{-2}\right]$ and $2 N A$ is the total membrane area $\left[\mathrm{m}^{2}\right]$.

\section{RED stack}

Cross-flow RED stacks (REDstack BV, the Netherlands) with an active area of $10 \times 10 \mathrm{~cm}^{2}$ were used in the experiments. The stack end-plates embedded mesh electrodes $\left(9.8 \times 9.8 \mathrm{~cm}^{2}\right)$ made of platinized titanium (MAGNETO Special Anodes BV, the Netherlands). Spacers with a woven netting (Saati PES 740/53, Saati S.p.A. Italy) and integrated silicone rubber gaskets (DEUKUM GmbH, Germany) were used to accommodate the feedwaters and seal the compartments. All stacks examined in the present study consisted of five cell pairs (five AEM and CEM pairs) plus an extra CEM to seal the electrolyte compartment. For the stack assembly, the chosen AEMs were: Fujifilm AEM type I and type 10 (FUJIFILM Manufacturing Europe BV, the Netherlands), Neosepta AMX-fg and the monovalent ion selective Neosepta ACS (ASTOM Corp. Ltd., Japan). Neosepta CMX-fg (ASTOM Corp. Ltd., Japan) was used as CEM to seal the electrolyte compartment of all stacks. The main properties of the membranes are compared in Table 1.

\section{Feedwaters}

Sodium chloride ( $\mathrm{NaCl}, 99.5 \%$ purity, ESCO, the Netherlands) solutions with $17 \mathrm{mM}$ and $508 \mathrm{mM}$ concentrations were used as artificial river water and seawater, respectively. To evaluate the effect of multivalent anions on AEMs in RED, sodium sulfate ( $\geq 99 \%$ purity, VWR Chemicals, Belgium) was added to the feedwaters in different configurations. The scenarios differed based on to which feedwater the sulfate was added (river, sea, or both) and to the fraction of sulfate $(10,25$, and $50 \mathrm{~mol} \%)$. Table 2 illustrates the sequence of feedwaters used in each stack experiment. The sulfate fractions were chosen to resemble the amounts found in natural salinity gradients. Rijnaarts et al. reported 11 mol.\% sulfate in seawater (Wadden Sea, the Netherlands) and $30 \mathrm{~mol} . \%$ sulfate in river water (IJsselmeer, the Netherlands) $)^{23}$. The choice of substituting equimolar amounts of $\mathrm{NaCl}$ with $\mathrm{Na}_{2} \mathrm{SO}_{4}$ is to maintain the extent of the chloride gradient constant when adding multivalent ions on both sides. Table 3 shows the ionic radii, hydration free energy, and $\mathrm{pK}_{\mathrm{a}}$ for selected monovalent and multivalent ions.

The electrode rinse solution was a mixture of $0.05 \mathrm{M}$ potassium hexacyanoferrate(II)/0.05 M potassium hexacyanoferrate(III) (both $\geq 96 \%$ purity, VWR Chemicals, Belgium). Sodium chloride (0.25 M) was added as a supporting electrolyte. Experiments were performed in the laboratory at $23.0 \pm 0.5^{\circ} \mathrm{C}$. Temperature and conductivity of the artificial feedwaters were measured before each experiment. The flow velocity was chosen to be $1 \mathrm{~cm} \mathrm{~s}^{-1}$. Fresh feedwaters were continuously supplied to the RED stacks, in a single-pass configuration, while the electrolyte was recirculated though the electrode compartments in a closed loop. For the long term ED experiment, the feedwaters (initially $0.1 \mathrm{M} 100 \mathrm{~mol} \% \mathrm{NaCl}$, then $50 \mathrm{~mol} . \%$ $\mathrm{Na}_{2} \mathrm{SO}_{4}$ ) were also recirculated. Peristaltic pumps (Masterflex L/S Digital drive, Cole-Palmer, USA) were used to pump the liquids through the hydraulic circuits. A diaphragm pressure control valve (KNF FDV 30, KNFVerder BV, the Netherlands) was used to keep the electrolyte at 0.3 bar overpressure to avoid bulging of the water compartments.

\section{Stack measurements}

All electrochemical measurements at the stack level were performed with an Iviumstat (IVIUM Technologies BV, the Netherlands). The IviumSoft Electrochemistry Software was used to control the potentiostat. For RED experiments, electrochemical measurements were continuously repeated in a loop for $8.5 \mathrm{~h}$. A loop cycle consisted of one constant current step $\left(10 \mathrm{~A} \mathrm{~m}^{-2}\right.$, in galvanostatic mode) and a chronopotentiometric series 
Table 3. Hydrated radii, hydration free energy, and $\mathrm{pK}_{\mathrm{a}}$ of selected monovalent and multivalent ions.

\begin{tabular}{lllll}
\hline Ion & lonic radius $[\mathrm{nm}]^{32}$ & Hydrated radius $[\mathrm{nm}]^{32}$ & Hydration free energy $\left.[\mathrm{kJ} \mathrm{mol}]^{-1}\right]^{32}$ & $\mathrm{pK}_{\mathrm{a}}[-]^{33}$ \\
\hline $\mathrm{Cl}^{-}$ & 0.181 & 0.332 & -363 & $(-)$ \\
$\mathrm{SO}_{4}^{2-}$ & 0.258 & 0.379 & -1145 & $1.99(2 \mathrm{nd})$ \\
$\mathrm{PO}_{3}^{2+}$ & 0.238 & 0.339 & $(-)$ & $2.16(1 \mathrm{st}), 7.21(2 \mathrm{nd}), 12.32(3 \mathrm{rd})$ \\
$\mathrm{CO}_{3}^{2-}$ & 0.156 & $(-)$ & $(-)$ & $6.4(1 \mathrm{st}), 10.3(2 \mathrm{nd})$ \\
$\mathrm{Na}^{+}$ & 0.117 & 0.358 & -365 & 14.8 \\
$\mathrm{Ca}^{2+}$ & 0.100 & 0.412 & -1504 & 12.6 \\
$\mathrm{Mg}^{2+}$ & 0.072 & 0.428 & -1828 & 11.4 \\
\hline
\end{tabular}

consisting of four current steps separated by open-circuit steps $(7.5,10$, 12.5 , and $15 \mathrm{~A} \mathrm{~m}^{-2}$ ), in galvanostatic mode. The duration of one loop cycle was $15 \mathrm{~min}$ ( $7.5 \mathrm{~min}$ constant current). The effluents from the sea and river water compartment were sampled during every other constant current step. The concentration of anions was measured with ion chromatography (IC) (Metrohm Compact IC Flex 930, Metrohm Nederland, the Netherlands). The open-circuit steps at the beginning of each chronopotentiometric series was used to evaluate the OCV. Stack electrical resistance was evaluated from the slope of the I-V curve obtained from the chronopotentiometric series, no blank correction was performed. Supporting Fig. 3 illustrates how the results from the time series of chronopotentiometry and different feedwaters were used to obtain the normalized parameters vs. sulfate fraction plots. For the ED experiment, a loop cycle consisted of one constant current step (10 $\mathrm{Am}^{-2}$, in galvanostatic mode) and two chronopotentiometric series consisting of four current steps separated by open-circuit steps $\left(7.5,10,12.5\right.$ and $\left.15 \mathrm{~A} \mathrm{~m}^{-2}\right)$, in galvanostatic mode. The duration of one loop cycle was $45 \mathrm{~min}$ ( $30 \mathrm{~min}$ constant current). Matlab software (The MathWorks Inc., USA) was used for data analysis. Matlab scripts were developed for the specific purpose of processing the data from the chronopotentiometric series. For ease of comparison, the results have been normalized to the values measured with clean waters (before introducing multivalent anions in the feedwaters). Stack experiments were performed at least in duplicate (except ED experiments).

\section{Membrane characterizations}

The membrane characterizations (water uptake, permselectivity, and membrane resistance) were performed based on procedures available in the literature ${ }^{31}$. For permselectivity measurement in presence of multivalent ions, the solution concentrations and voltages used in the experiment and calculations were the ones after uphill transport (as predicted by the model; further details of the compositions in Supporting Table 3). Equilibration of the membranes prior to permselectivity measurements was carried out in the $0.1 \mathrm{M}$ solution containing amounts of sodium chloride and sulfate corresponding to the equilibrium after uphill transport (further details of the compositions in Supporting Table 3). The composition of the solutions for the permselectivity measurement was chosen to avoid uphill transport as that would introduce an undesired potential loss, which would lead to a wrong measurement. Electrical resistance and permselectivity measurements were performed with an Autolab PGSTAT30 (Metrohm Nederland, the Netherlands). The NOVA 2.1.3 software was used to control the potentiostat. Membrane characterizations were performed at least in triplicate.

\section{DATA AVAILABILITY}

The data that support the findings of this study are available from the corresponding author upon reasonable request.

Received: 8 November 2019; Accepted: 27 April 2020; Published online: 15 June 2020

\section{REFERENCES}

1. Logan, B. E. \& Elimelech, M. Membrane-based processes for sustainable power generation using water. Nature 488, 313-319 (2012).
2. Post, J. W. et al. Salinity-gradient power: evaluation of pressure-retarded osmosis and reverse electrodialysis. J. Memb. Sci. 288, 218-230 (2007).

3. Li, W. et al. A novel hybrid process of reverse electrodialysis and reverse osmosis for low energy seawater desalination and brine management. Appl. Energy 104, 592-602 (2013).

4. Tedesco, M. et al. Reverse electrodialysis with saline waters and concentrated brines: a laboratory investigation towards technology scale-up. J. Memb. Sci. 492, 9-20 (2015).

5. Tedesco, M., Cipollina, A., Tamburini, A. \& Micale, G. Towards 1 kW power production in a reverse electrodialysis pilot plant with saline waters and concentrated brines. J. Memb. Sci. 522, 226-236 (2017).

6. Tamburini, A. et al. Reverse electrodialysis heat engine for sustainable power production. Appl. Energy 206, 1334-1353 (2017).

7. Ortega-Delgado, B. et al. Reverse electrodialysis heat engine with multi-effect distillation: exergy analysis and perspectives. Energy Convers. Manag. 194, 140-159 (2019).

8. Giacalone, F. et al. Thermolytic reverse electrodialysis heat engine: model development, integration and performance analysis. Energy Convers. Manag. 189, 1-13 (2019).

9. Giacalone, F. et al. The first operating thermolytic reverse electrodialysis heat engine. J. Memb. Sci. 595, 1-12 (2020).

10. Kingsbury, R. S., Chu, K. \& Coronell, O. Energy storage by reversible electrodialysis: the concentration battery. J. Memb. Sci. 495, 502-516 (2015).

11. van Egmond, W. J. et al. Performance of an environmentally benign acid base flow battery at high energy density. Int. J. Energy Res. 42, 1524-1535 (2018).

12. Post, J. W., Hamelers, H. V. M. \& Buisman, C. J. N. Energy recovery from controlled mixing salt and fresh water with a reverse electrodialysis system. Environ. Sci. Technol. 42, 5785-5790 (2008).

13. Galama, A. H., Post, J. W., Hamelers, H. V. M., Nikonenko, V. V. \& Biesheuvel, P. M. On the origin of the membrane potential arising across densely charged ion exchange membranes: how well does the teorell-meyer-sievers theory work? J. Membr. Sci. Res. 2, 128-140 (2016).

14. Veerman, J., Saakes, M., Metz, S. J. \& Harmsen, G. J. Reverse electrodialysis: evaluation of suitable electrode systems. J. Appl. Electrochem. 40, 1461-1474 (2010).

15. Vermaas, D. A., Kunteng, D., Saakes, M. \& Nijmeijer, K. Fouling in reverse electrodialysis under natural conditions. Water Res. 47, 1289-1298 (2013).

16. Post, J. W. J. W., Hamelers, H. V. M. H. V. M. \& Buisman, C. J. N. C. J. N. Influence of multivalent ions on power production from mixing salt and fresh water with a reverse electrodialysis system. J. Memb. Sci. 330, 65-72 (2009).

17. Higa, M., Tanioka, A. \& Miyasaka, K. Simulation of the transport of ions against their concentration gradient across charged membranes. J. Memb. Sci. 37, 251-266 (1988).

18. Vermaas, D. A. D. A., Veerman, J., Saakes, M. \& Nijmeijer, K. Influence of multivalent ions on renewable energy generation in reverse electrodialysis. Energy Environ. Sci. 7, 1434-1445 (2014).

19. Lacey, R. E. Energy by reverse electrodialysis. Ocean Eng. 7, 1-47 (1980).

20. Badessa, T. \& Shaposhnik, V. The electrodialysis of electrolyte solutions of multicharged cations. J. Memb. Sci. 498, 86-93 (2016).

21. Moreno, J., Díez, V., Saakes, M. \& Nijmeijer, K. Mitigation of the effects of multivalent ion transport in reverse electrodialysis. J. Memb. Sci. 550, 155-162 (2018).

22. Rijnaarts, T., Huerta, E., Van Baak, W. \& Nijmeijer, K. Effect of divalent cations on red performance and cation exchange membrane selection to enhance power densities. Environ. Sci. Technol. 51, 13028-13035 (2017).

23. Rijnaarts, T., Moreno, J., Saakes, M., de Vos, W. M. \& Nijmeijer, K. Role of anion exchange membrane fouling in reverse electrodialysis using natural feed waters. Colloids Surf. A Physicochem. Eng. Asp. 560, 198-204 (2019). 
24. Nightingale, E. R. Jr. Phenomenological theory of ion solvation. Effective radii of hydrated ions. J. Phys. Chem. 63, 1381-1387 (1959).

25. Luo, T., Abdu, S. \& Wessling, M. Selectivity of ion exchange membranes: a review. J. Memb. Sci. 555, 429-454 (2018).

26. Epsztein, R., Shaulsky, E., Qin, M. \& Elimelech, M. Activation behavior for ion permeation in ion-exchange membranes: Role of ion dehydration in selective transport. J. Memb. Sci. 580, 316-326 (2019).

27. Liao, J. et al. Amphoteric ion-exchange membranes with superior mono-/bi-valent anion separation performance for electrodialysis applications. J. Memb. Sci. 577, 153-164 (2019).

28. Paltrinieri, L. et al. Hybrid polyelectrolyte-anion exchange membrane and its interaction with phosphate. React. Funct. Polym. 133, 126-135 (2018).

29. Weinstein, J. N. \& Leitz, F. B. Electric power from differences in salinity: the dialytic battery. Sci. (80-). 191, 557-559 (1976).

30. Veerman, J., Saakes, M., Metz, S. J. \& Harmsen, G. J. Reverse electrodialysis: a validated process model for design and optimization. Chem. Eng. J. 166, 256-268 (2011).

31. Długołecki, P., Nymeijer, K., Metz, S. \& Wessling, M. Current status of ion exchange membranes for power generation from salinity gradients. J. Memb. Sci. 319, 214-222 (2008).

32. Tansel, B. Significance of thermodynamic and physical characteristics on permeation of ions during membrane separation: Hydrated radius, hydration free energy and viscous effects. Sep. Purif. Technol. 86, 119-126 (2012).

33. Haynes, W. M. CRC handbook of chemistry and physics. 97th ed. (CRC Press, 2016)

\section{ACKNOWLEDGEMENTS}

This work was performed in the cooperation framework of Wetsus, European Centre of Excellence for Sustainable Water Technology (www.wetsus.eu). Wetsus is cofunded by the Dutch Ministry of Economic Affairs and Ministry of Infrastructure and Environment, the Province of Fryslân, and the Northern Netherlands Provinces. This project has also received funding from the European Union's Horizon 2020 research and innovation program under the Marie Skłodowska-Curie grant agreement No. 665874. The authors would like to thank the participants of the research theme "Blue Energy" for their input and suggestions and their financial support.

\section{AUTHOR CONTRIBUTIONS}

DP, MS, ZB, and KN conceived the idea and experiments for the presented study. DP and CC carried out the experimental work, model calculations, and raw data analysis. MS encouraged DP to investigate the longer-term effect of sulfate exposure on membrane electrical resistance. $\mathrm{MS}, \mathrm{ZB}$, and $\mathrm{KN}$ supervised the findings of this work, providing critical feedback and helping shape the research, analysis, and manuscript. All authors discussed the results and contributed to the manuscript.

\section{COMPETING INTERESTS}

The authors declare no competing interests.

\section{ADDITIONAL INFORMATION}

Supplementary information is available for this paper at https://doi.org/10.1038/ s41545-020-0073-7.

Correspondence and requests for materials should be addressed to K.N.

Reprints and permission information is available at http://www.nature.com/ reprints

Publisher's note Springer Nature remains neutral with regard to jurisdictional claims in published maps and institutional affiliations.

\section{(c) (1)}

Open Access This article is licensed under a Creative Commons Attribution 4.0 International License, which permits use, sharing, adaptation, distribution and reproduction in any medium or format, as long as you give appropriate credit to the original author(s) and the source, provide a link to the Creative Commons license, and indicate if changes were made. The images or other third party material in this article are included in the article's Creative Commons license, unless indicated otherwise in a credit line to the material. If material is not included in the article's Creative Commons license and your intended use is not permitted by statutory regulation or exceeds the permitted use, you will need to obtain permission directly from the copyright holder. To view a copy of this license, visit http://creativecommons. org/licenses/by/4.0/.

(c) The Author(s) 2020 